\title{
miR-30a-5p in the tumorigenesis of renal cell carcinoma: A tumor suppressive microRNA
}

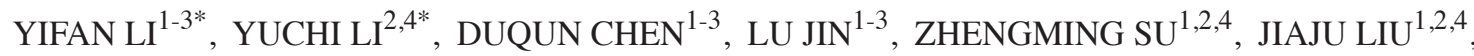 \\ HONGFANG DUAN ${ }^{5}$, XIAOQING $\mathrm{LI}^{4}$, ZHENGYU QI ${ }^{2}$, MIN SHI $^{2}$, LIANGCHAO NI $^{1,2}$, SHANGQI YANG ${ }^{1,2}$, \\ YAOTING GUI ${ }^{2}$, XIANGMING MAO ${ }^{1,2},{\text { YUN } \mathrm{CHEN}^{6} \text { and YONGQING LAI }}^{1,2}$ \\ ${ }^{1}$ Department of Urology, Peking University Shenzhen Hospital, Shenzhen, Guangdong 518036; \\ ${ }^{2}$ The Guangdong and Shenzhen Key Laboratory of Male Reproductive Medicine and Genetics, \\ Peking University Shenzhen Hospital, Institute of Urology of Shenzhen PKU-HKUST Medical Center, Shenzhen, \\ Guangdong 518036; ${ }^{3}$ Department of Urology, Anhui Medical University, Hefei, Anhui 230032; ${ }^{4}$ Department of Urology, \\ Shantou University Medical College, Shantou, Guangdong 515041; ${ }^{5}$ Department of Otolaryngological, \\ Guangzhou Medical University, Guangzhou, Guangdong 510000; ${ }^{6}$ Department of Ultrasound Division, \\ Peking University Shenzhen Hospital, Shenzhen, Guangdong 518036, P.R. China
}

Received April 25, 2015; Accepted March 7, 2016

DOI: $10.3892 / \mathrm{mmr} .2016 .5024$

\begin{abstract}
Renal cell carcinoma (RCC) is the most common type of malignant tumor of the adult kidney and has a poor prognosis. MicroRNAs (miRs) are important in a wide range of biological and pathological processes, including cell differentiation, migration, growth, proliferation, apoptosis and metabolism. The present study aimed to determine the role exerted by miR-30a-5p in the tumorigenesis of RCC. The expression levels of miR-30a-5p in RCC tissues and RCC-derived cells were demonstrated to be significantly downregulated by real-time quantitative polymerase chain reaction (RT-qPCR). Wound scratch assay, cell proliferation assay and flow cytometric analysis revealed that the abilities of migration and proliferation of the RCC-derived cells were suppressed, whereas cell apoptosis was promoted, when miR-30a-5p was overexpressed in these cells. N-acetylgalactos aminyltransferase 7 (GALNT7) was predicted to be one target gene of miR-30a-5p by bioinformatics analysis. Luciferase reporter assay, RT-qPCR and western blotting were performed
\end{abstract}

Correspondence to: Professor Yongqing Lai, Department of Urology, Peking University Shenzhen Hospital, 1120 Lianhua Road, Shenzhen, Guangdong 518036, P.R. China

E-mail: yqlord@163.com

Professor Yun Chen, Department of Ultrasound Division, Peking University Shenzhen Hospital, 1120 Lianhua Road, Shenzhen Guangdong 518036, P.R. China

E-mail: cyun126@126.com

*Contributed equally

Key words: renal cell carcinoma, microRNA-30a-5p, polypeptide $\mathrm{N}$-acetylgalactosaminyltransferase 7 , tumor suppressor to confirm that GALNT7 is the direct conserved target of miR-30a-5p. These results suggested that miR-30a-5p has a tumor-suppressive role in the tumorigenesis of RCC.

\section{Introduction}

Renal cell carcinoma (RCC), arising from the renal cortex, is the most common type of malignant tumor in the adult kidney (1). Clear cell RCC is the most common histological type of RCC, and is more aggressive than other types (2). Novel target therapeutic strategies have improved the treatment of patients with $\mathrm{RCC}$, although radical nephrectomy remains the predominant treatment strategy for patients with RCC due to the resistance of the tumor to radiation and chemotherapy $(3,4)$. However, early detection and diagnosis of RCC is difficult due to the lack of clinical signs and manifestations (3). Additionally, RCC is highly vascular, invasive and metastatic. Once metastasis occurs, patients with RCC have a poorer prognosis. A third of patients with RCC develop distant metastasis at initial diagnosis, and $\sim 50 \%$ of these patients develop disease recurrence, of which two thirds recur within the first year $(4,5)$. Thus, it is crucial to investigate the mechanism underlying RCC tumorigenesis.

MicroRNAs (miRNAs) are a class of noncoding RNA, typically 20-23 nt in length, which are cleaved from 70-100 nt hairpin-shaped precursors (pre-miRNA) (6). Several studies have demonstrated that miRNAs are important in a wide range of biological and pathological processes, including cell differentiation, migration, growth, proliferation, apoptosis and metabolism (6-9). A link between miRNA function and cancer pathogenesis has been supported by studies examining the signatures and functions of miRNA in clinical samples (10). In urological cancer, including RCC, the aberrant expression of miRNAs has been reported between malignant and normal renal tissues, and a specific miRNAs have been found to exert novel oncogenic or tumor suppressive functions though target genes $(11,12)$. 
Previous studies have identified that downregulated miR-30a and miR-30a-3p increase angiogenesis-specific Delta-like ligand 4 (DLL4) and hypoxia-inducible factor $2 \alpha$ (HIF $2 \alpha$ ) expression, and promote tumor metastasis and growth, in clear cell RCC $(13,14)$. Previous studies on expression have shown that miR-30a-5p is downregulated in malignancies of the lung (14), blood (15), liver (16), nasopharynx (17), bone (18), breast (19) and bladder (20). However, miR-30a-5p has been reported to be upregulated in glioma $(21,22)$ cell lines and specimens, compared with normal tissues and cell lines. In RCC, several microarray chip assays have revealed consistent results, that the expression levels of miR-30a-5p are lower in RCC tissues and metastatic RCC tissues, compared with adjacent normal tissues and paired primary RCC tissues (24-28). These data suggest that miR-30a-5p may offer potential as a useful diagnostic, prognostic and predictive biomarker in the treatment of RCC. However, the expression, function and molecular mechanism of miR-30a-5p in RCC remain to be fully elucidated.

In the present study, the expression levels of miR-30a-5p in RCC specimens and cell lines were examined, and the function of miR-30a-5p in RCC cells was investigated using cell migration, proliferation and apoptotic assays. To further examine the miR-30a-5p-mediated molecular pathway, polypeptide $\mathrm{N}-\mathrm{ac}$ etylgalactosaminyltransferase 7 (GALNT7) was identified as a potential target of miR-30a-5p. Collectively, the results of the present study may determine whether miR-30a-5p has an inhibitory effect in the carcinogenesis of RCC though changes in the expression of GALNT7.

\section{Materials and methods}

Cell lines and transfection. The human RCC cell lines (786-O and ACHN), human cervical cancer cell line (Hela) and human embryo kidney cell line (293T) were obtained from American Type Culture Collection (Manassas, VA, USA), and were routinely cultured in Dulbecco's modified Eagle's medium (DMEM)/high glucose medium (Gibco; Thermo Fisher Scientific, Inc., Waltham, MA, USA) supplemented with $10 \%$ fetal bovine serum (FBS; Gibco; Thermo Fisher Scientific, Inc.), $1 \%$ antibiotics $(100 \mathrm{U} / \mathrm{ml}$ penicillin and $100 \mathrm{mg} / \mathrm{ml}$ streptomycin; Gibco; Thermo Fisher Scientific, Inc.) and $1 \%$ glutamate (Gibco; Thermo Fisher Scientific, Inc.). All cells were cultured at $37^{\circ} \mathrm{C}$ in a humidified chamber containing $5 \% \mathrm{CO}_{2}$.

To induce the upregulation of miR-30a-5p in the cells, chemically synthesized miR-30a-5p mimics (GenePharma, Inc., Shanghai, China) were transfected into the cells at a confluence of 60-70\% using Lipofectamine 2000 (Invitrogen; Thermo Fisher Scientific, Inc.), which were mixed in Opti-MEM ${ }^{\circledR}$ I reduced serum medium (Gibco; Thermo Fisher Scientific, Inc.) following culture for $24 \mathrm{~h}$. To validate the transient transfection efficiency, fluorescence microscopy (using a DMIRB fluorescence microscope; Leica Microsystems, Wetzlar, Germany) was performed after transfection of Negative Control-Fluorescein amidite (NC-FAM), and real-time quantitative polymerase chain reaction (RT-qPCR) was performed after the transfection of miR-30a-5p mimics and NC. The sequences of the miRNAs (NC-FAM, NC and miR-30a-5p mimic) are summarized in Table I.
Tissue collection. The collection and use of tissue samples in the present study were reviewed and approved by the ethics committee of Peking University Shenzhen Hospital (Shenzhen, Guangdong, China). Written informed consent were signed and obtained from all 32 patients. In the present study, a total of 32 paired fresh RCC and adjacent normal tissue samples (located $5.0 \mathrm{~cm}$ from the visible RCC lesions) were obtained from Peking University Shenzhen Hospital (Shenzhen, China). Once dissected, all fresh tissue samples were immediately immersed in RNAlater (Qiagen, Hilden, Germany) and frozen in liquid nitrogen for total RNA extraction. The clinicopathological information obtained from the patients is shown in Table II. Stage classification was performed using the 2010 American Joint Committee on Cancer staging system (29).

Total RNA extraction, reverse transcription and RT-qPCR. The 786-O, ACHN and $293 \mathrm{~T}$ cells $\left(4 \times 10^{5}\right.$ cells/well) were plated into 6-well plates (BD Biosciences, San Jose, CA, USA) with three replicate wells, respectively. After $24 \mathrm{~h}$ at $37^{\circ} \mathrm{C}$, the cells were trypsinized to extract the total RNA using TRIzol ${ }^{\circledR}$ reagent (Invitrogen; Thermo Fisher Scientific, Inc.). Total RNA was extracted from the 32 paired RCC tissues and normal tissues using TRIzol ${ }^{\circledR}$ reagents and were purified using the RNeasy ${ }^{\circledR}$ Maxi kit (Qiagen), according to the manufacturer's protocol. For the homogenization step, 50-100 mg tissue or $\sim 1.5 \times 10^{6}$ cells were added to $1 \mathrm{ml}$ RNAiso Plus reagent and homogenized using a motor-driven tissue grinder (OSE-Y10; Tiangen Biotech (Beijing) Co., Ltd., Beijing, China). Subsequently, the homogenized samples were incubated with RNAiso Plus reagent for $5 \mathrm{~min}$ at room temperature to permit the complete dissociation of nucleoprotein complexes. The tubes were agitated vigorously by hand for $15 \mathrm{sec}$, and then the samples were centrifuged at $12,000 \mathrm{x}$ g for $15 \mathrm{~min}$ at $4^{\circ} \mathrm{C}$.

The RNA samples with 260/280 ratios of 1.8-2.0 were used in the subsequent experiments. The total RNA was converted into a cDNA template using an miScript II RT kit (Qiagen) for quantification of miR-30a-5p, or a PrimeScript ${ }^{\mathrm{TM}} \mathrm{RT}$ reagent kit (Takara Bio., Inc., Otsu, Japan) for quantification of GALNT7 mRNA.

The expression of miR-30a-5p was confirmed using the miScriptSYBR ${ }^{\circledR}$ green PCR kit (Qiagen), and the mRNA expression of GALNT7 was validated using SYBR ${ }^{\circledR}$ Premix Ex $\mathrm{Taq}^{\mathrm{TM}}$ II (Takara Bio, Inc.), respectively, on a Roche lightcycler 480 Real-Time PCR system (Roche Applied Science, Mannheim, Germany). The $20 \mu 1$ reaction mixture contained $10 \mu 1$ 2X QuantiTect SYBR Green PCR Master mix, $2 \mu 1$ 10X miScript Universal Primer, $0.4 \mu 1$ specific miRNA primer, $1 \mu \mathrm{l}$ cDNA template and $6.6 \mu 1$ RNase-free water. The thermocycling steps were $95^{\circ} \mathrm{C}$ for $15 \mathrm{~min}$, followed by 40 cycles at $94^{\circ} \mathrm{C}$ for $15 \mathrm{sec}, 55^{\circ} \mathrm{C}$ for $30 \mathrm{sec}$ and $72^{\circ} \mathrm{C}$ for 30 sec. U6 and GAPDH were used as internal controls. The expression levels were calculated as fold changes relative to the U6 and GAPDH controls, which was based on the following equation: Relative expression $=2^{-\Delta \Delta C q}(30)$. The primers used are shown in Table I.

Wound scratch assay. Cell migration ability was examined using a wound scratch assay. The cells $\left(\sim 3 \times 10^{5}\right.$ cells/well $)$ were seeded into a 12 -well dish and, on reaching $80-90 \%$ confluence, the miR-30a-5p mimic or negative control (NC) 
Table I. Sequences of primers and microRNAs.

\begin{tabular}{ll}
\hline Primer/microRNA & \multicolumn{1}{c}{ Sequence } \\
\hline GALNT7 & Forward 5'-GGGATTATTTGCCATTGAACGA-3' \\
GAPDH & Reverse 5'-AGACGGTAGATATGTCCAACAC-3' \\
miR-30a-5p & Forward 5'-GGAGCGAGATCCCTCCAAAAT-3' \\
& Reverse 5'-GGCTGTTGTCATACTTCTCATGG-3' \\
U6 & Forward 5'-TGTAAACATCCTCGACTGGAAG-3' \\
miR-30a-5p mimic & Reverse provided by the miScript SYBR ${ }^{\circledR}$ Green \\
NC & PCR kit ('iagen) \\
NC-FAM & Forward 5'-CTCGCTTCGGCAGCACA-3' \\
& Reverse 5'-ACGCTTCACGAATTTGCGT-3' \\
& Forward5'-UGUAAACAUCCUCGACUGGAAG-3' \\
& Reverse 5'-UCCAGUCGAGGAUGUUUACAUU-3' \\
& Forward 5'-UUCUCCGAACGUGUCACGUTT-3' \\
& Reverse 5'-ACGUGACACGUUCGGAGAATT-3'
\end{tabular}

GALNT7, polypeptide N-acetylgalactosaminyltransferase 7; miR, microRNA; NC, negative control; NC-FAM; NC-fluorescein amino-modified oligonucleotide.

were introduced into the 786-O and ACHN cells using Lipofectamine 2000 (Invitrogen; Thermo Fisher Scientific, Inc.). The NC (GenePharma, Inc., Shanghai, China) comprised miRNA oligonucleotides that were chemically synthesized in order to eliminate the non-sequence binding effects. The sequences of the miRNAs (NC, NC-FAM and miR-30a-5p mimic) were all summarized in Table I. Following $6 \mathrm{~h}$ of transfection, a sterile $200 \mu \mathrm{l}$ pipette tip and markers were used to introduce a scratch into the cell monolayer. The cells were then rinsed with phosphate-buffered saline (PBS) three times, cultured in DMEM, and incubated at $37^{\circ} \mathrm{C}$. Images of the scratches were acquired using a digital camera system (model DMIRB; Leica Microsystems, Wetzlar, Germany) at 0 and $24 \mathrm{~h}$ following introduction of the scratches at the same points. The experiments were performed in three independent repeats in triplicate, and analyzed in a double-blinded manner by at least two observers.

Cell proliferation assay. A 3-(4,5-dimethylthiazol-2-yl)-2,5-diphenyltetrazoliumbromide (MTT; $5 \mathrm{mg} / \mathrm{ml}$; Sigma-Aldrich, St. Louis, MO, USA) assay was performed to determine the proliferation abilities of the 786-O and ACHN cells. The 786-O and ACHN cells were seeded into 96-well plates at a cell density of $5 \times 10^{3}$ cells/well, with five replicate wells for each condition. After $24 \mathrm{~h}$, the cells in each wells were transfected with either 5 pmol miR-30a-5p mimic or NC. MTT (20 $\mu \mathrm{l})$ was added to each well and the plate was incubated at $37^{\circ} \mathrm{C}$ for $\sim 5 \mathrm{~h}$ prior to measurement. The MTT medium mixtures were then discarded and the reaction was terminated by the addition of $120 \mu \mathrm{l}$ dimethylsulphoxide (Sigma-Aldrich). Following agitation for $15 \mathrm{~min}$ at room temperature, the optical density was measured at the wavelength of $490 \mathrm{~nm}$, with $630 \mathrm{~nm}$ as the reference wavelength, using an enzyme immunoassay
Table II. Clinicopathological features of patients with renal cell carcinoma.

\begin{tabular}{lc}
\hline Characteristic & Cases (n) \\
\hline Mean age; range (years) & $52 ; 29-71$ \\
Gender & \\
Male/female & $20 / 12$ \\
Histological type & $29 / 3$ \\
Clear cell/papillary & \\
Primary tumor stage & $18 / 13 / 1$ \\
T1/T2/T3+T4 & \\
Fuhrman grade & $11 / 15 / 4 / 2$ \\
I/II/III/IV & \\
AJCC clinical stage & $17 / 14 / 1$ \\
I/II/III+IV & \\
\hline
\end{tabular}

AJCC, American Joint Committee on Cancer.

instrument (Bio-Rad Laboratories, Inc., Hercules, CA, USA). All assays were performed in triplicate.

Flow cytometric analysis. The early apoptotic rates of the 786-O and ACHN cells were stained with fluorescein isothiocyanate (FITC)-conjugated Annexin V (Annexin V-FITC) and propidium iodide (PI; Invitrogen; Thermo Fisher Scientific, Inc.) and quantified using flow cytometry (EPICS, Xl-4, Beckman Coulter, Brea, CA, USA). The 786-O and ACHN cells $\left(3 \times 10^{5}\right)$ were seeded into 6 -well plates, and 200 pmol of either miR-30a-5p mimic or NC were introduced to the 
cells via transfection for $6 \mathrm{~h}$. Subsequently, the cells were incubated at $37^{\circ} \mathrm{C}$ in a humidified chamber containing $5 \% \mathrm{CO}_{2}$ for $48 \mathrm{~h}$ following transfection. The cells, including floating cells, were collected and washed twice with pre-chilled PBS. The cells were resuspended in $100 \mu 11 \mathrm{X}$ binding buffer (comprising $10 \mathrm{mM}$ HEPES, $140 \mathrm{mM} \mathrm{NaCl}, 2.5 \mathrm{mM} \mathrm{CaCl}$, pH 7.4; Invitrogen; Thermo Fisher Scientific, Inc.) and stained with $5 \mu \mathrm{l}$ Annexin V-FITC and PI for $15 \mathrm{~min}$ at room temperature, using an Annexin V-FITC/PI detection kit (Invitrogen; Thermo Fisher Scientific, Inc.). The flow cytometric measurements were obtained within $30 \mathrm{~min}$ of staining, and $400 \mu \mathrm{l}$ of $1 \mathrm{X}$ binding buffer was added to each sample upon measurement. Each experiment was performed at least three times.

Bioinformatics analysis. Computational algorithms have been confirmed as an effective method for predicting the candidate target genes of a specific miRNA. In the present study, the following target prediction algorithms were used: miRanda (http://mirdb.org/miRDB/index.html), TargetScan Release 6.2 (http://www.targetscan.org), microRNA (http://www. microrna.org) and miRWalk (http://www.umm.uni-heidelberg. de/apps/zmf/mirwalk). These approaches are based on the identification of elements in the 3'-untranslated region (UTR) of target genes, which are complementary to the seed sequence of the microRNA of interest. Putative genes predicted by at least three algorithms were accepted, and candidates were selected based on the gene function.

Luciferase reporter assay. The 3'-UTR of GALNT7 (519 bp), containing the putative binding site (5'-TGTTTAC-3') for miR-30a-5p, was cloned into an empty psiCHECK-2 Vector (Promega, Madison, WI, USA), generating the wild-type psiCHECK2-3'-UTR. The primer sequences for the 3'-UTR of GALNT7 containing the miR-30a-5p binding site (forward primer, 5'-CCGCTCGAGCTACTGACAAGTAAATTTATAC AGG-3' and reverse primer, 5'-AAGGAAAAAAGCGGC CGCAGAGGCACTAAATGTGTTGA-3') were designed. The mutant type was generated by changing the putative binding site to 5'-ACAAATG-3' in the complementary site for seed region of miR-30a-5p. All constructed plasmids were verified by DNA sequencing analysis. The DNA sequencing analysis was performed on an Applied Biosystems 3730XL DNA Analyzer (Invitrogen; Thermo Fisher Scientific, Inc.) using the dideoxy-mediated chain-termination method.

Sequencing primers and the bacteria which contained the wild/mutant types of constructed plasmids were obtained from Thermo Fisher Scientific, Inc. The detected DNA sequences were also obtained from Thermo Fisher Scientific, Inc., and the DNA sequences were compared with the sequences designed for the present study to check whether the sequences, including the 3'-UTR of GALNT7 (wide or mutant type), had been incorporated into the psi-CHECK2 vector.

For the luciferase reporter assay, 293T and Hela cells were seeded into 24 -well plates, and were co-transfected with $0.5 \mu \mathrm{g}$ of the constructed plasmids and $40 \mathrm{pmol}$ of the miR-30a-5p mimic or NC using Lipofectamine 2000 (Invitrogen; Thermo Fisher Scientific, Inc.). At $24 \mathrm{~h}$ post-transfection, firefly and Renilla luciferase in the cells were detected using a Dual-Luciferase Reporter Assay system (Promega) on a Modulus ${ }^{\mathrm{TM}}$ Single Tube Multimode Reader (Turner
BioSystems, Madison, WI, USA). All experiments were performed in triplicate wells and repeated at least three times.

Western blot analysis. The 786-O and ACHN cells $\left(3 \times 10^{5}\right)$ were seeded into 6-well plates and transfected with 200 pmol of either the miR-30a-5p mimic or NC using Lipofectamine 2000 (Invitrogen; Thermo Fisher Scientific, Inc.) for 6 h. At 48 h post-transfection, radioimmunoprecipitation assay lysis buffer (Sigma-Aldrich) was used to lyse the cells on ice, and protein concentrations were quantified using a Pierce BCA Protein Assay kit (Thermo Fisher Scientific, Inc.). A total of $60 \mu \mathrm{g}$ of protein sample was loaded in each well and separated using $10 \%$ sodium dodecyl sulfate polyacrylamide gel electrophoresis (SDS-PAGE), with gels that were made prior to use: The formulation of the lower $10 \%$ separating gel was $4.0 \mathrm{ml} \mathrm{H}_{2} \mathrm{O}, 3.3 \mathrm{ml} 30 \%$ acrylamide, $2.5 \mathrm{ml} 1.5 \mathrm{M}$ Tris (pH 8.8), $0.1 \mathrm{ml} \mathrm{10 \%} \mathrm{SDS,} 0.1 \mathrm{ml} \mathrm{10 \%} \mathrm{AP,} 4 \mu 1$ tetramethylethylenediamine (TEMED), and that of the upper SDS-PAGE gel (5\% stacking gel) was: $2.7 \mathrm{ml} \mathrm{H}_{2} \mathrm{O}, 0.67 \mathrm{ml} \mathrm{30 \%}$ acrylamide, $0.5 \mathrm{ml} 1 \mathrm{MTris}(\mathrm{pH} 6.8), 0.04 \mathrm{ml} 10 \% \mathrm{SDS}, 0.04 \mathrm{ml} 10 \% \mathrm{AP}$ and $4 \mu 1$ TEMED. Subsequently, the proteins were transferred onto polyvinylidene difluoride (PVDF) membranes (EMD Millipore, Billerica, MA, USA) via wet blotting. The PVDF membranes were blocked with $5 \%$ nonfat milk at room temperature for $2 \mathrm{~h}$, and incubated with primary antibodies overnight at $4{ }^{\circ} \mathrm{C}$. The primary antibodies against GALNT7 and $\beta$-actin (internal control) were rabbit polyclonal anti-GALNT7 (1:1,000; cat. no. ab97645; Abcam, Cambridge, MA, USA) and rabbit polyclonal anti- $\beta$-actin $(1: 10,000$; cat. no. NB600-532; Novus Biologicals, Littleton, CO, USA), respectively. The second day, following washing three times with Tris-buffered saline with $0.05 \%$ Tween (TBST), the membranes were treated with horesradish peroxidase (HRP)-linked goat anti-rabbit IgG (1:10,000; cat. no. E031120-01, EarthOx, Life Sciences, Millbrae, CA, USA) for $2 \mathrm{~h}$, followed by the detection of protein bands using an Immun-Star ${ }^{\mathrm{TM}}$ HRP Chemiluminescence kit (Bio-Rad Laboratories, Inc.) Each assay was repeated at least three times.

Statistical analysis. The data are presented as the mean \pm standard deviation from three independent experiments. All data were analyzed using SPSS 19.0 statistical software (IBM SPSS, Inc., Armonk, NY, USA). The MTT data were analyzed using one-way analysis of variance. The clinicopathological information of the patients were analyzed using a $\chi^{2}$ test, whereas other data were analyzed using Student's $t$-test. $\mathrm{P}<0.05$ was considered to indicate a statistically significant difference.

\section{Results}

Expression levels of miR-30a-5p are downregulated in human RCC clinical specimens and cell lines. The expression levels of miR-30a-5p were examined in 32 paired RCC tissues and adjacent normal tissues, and the expression of miR-30a-5p was found to be significantly downregulated in the RCC tissues, compared with the adjacent normal tissues, as shown in Fig. 1A. The expression levels of miR-30a-5p in 293T cells and the three RCC cell lines were also quantified using RT-qPCR. As shown in Fig. 1B, the expression levels of miR-30a-5p were lower in the 786-O, ACHN and 769P cells $(\mathrm{P}<0.01)$, compared 
with the level in the $293 \mathrm{~T}$ cells, which was in accordance with the expression pattern of miR-30a-5p in the clinical specimens. The present study selected 786-O and ACHN cells to perform the subsequent functional experiments.

Transfection efficiency validation. The NC-fluorescein amino-modified oligonucleotide (NC-FAM), miR-30a-5p mimic or $\mathrm{NC}$ were transfected into $786-\mathrm{O}$ and $\mathrm{ACHN}$ to validate the transfection efficiency. As seen in Fig. 2A, transfection efficiency was $>90 \%$ when the cells were transfected with NC-FAM. RT-qPCR was also used to verify transfection efficiency, and it was revealed that miR-30a-5p was significantly overexpressed following transfection with the miR-30a-5p mimic in the 786-O $(\mathrm{P}<0.01)$ and $\mathrm{ACHN}(\mathrm{P}=0.015)$ cells (Fig. 2B).

miR-30a-5p suppresses RCC cell migration. The wound scratch assay showed that cell migration was significantly inhibited in the groups transfected with miR-30a-5p, compared with the NC groups. The inhibition rates of migration were $63.80 \%$ for the 786-O cells (Fig. 3A) and $67.95 \%$ for the ACHN cells (Fig. 3B), indicating that miR-30a-5p had a significant negative effect on RCC cell migration.

Proliferation of RCC cells is inhibited by miR-30a-5p mimic transfection. An MTT assay was performed to observe whether differential expression of miR-30a-5p affected the proliferation ability of the RCC cells. The results revealed that cell proliferation rates in the miR-30a-5p transfectants were significantly decreased by $4.66 \%$ ( $24 \mathrm{~h} ; \mathrm{P}<0.01), 10.99 \%$ (48 h; $\mathrm{P}<0.01)$ and $21.02 \%$ (72 $\mathrm{h} ; \mathrm{P}<0.01)$ in the 786-O cells (Fig. 4A). In the ACHN cells, the rates of proliferation inhibition were 6.84, 14.05 and $22.67 \%$ at 24,48 and $72 \mathrm{~h}$, respectively $(\mathrm{P}<0.01$; Fig. $4 \mathrm{~B})$.

Cell apoptosis is induced by miR-30a-5p mimic. To investigate the effect of miR-30a-5p on the apoptosis of RCC cells, flow cytometry was performed to detect the early apoptotic rate. The results showed that, compared with the cells transfected with $\mathrm{NC}$, the apoptotic rate was significantly increased in the miR-30a-5p mimic-transfected $786-\mathrm{O}$ cells $(2.94$, vs. $11.50 \%$, respectively; $\mathrm{P}<0.01$; Fig. 5A) and the miR-30a-5p mimic-transfected ACHN cells (2.00, vs. $17.90 \%$, respectively; $\mathrm{P}<0.01$, Fig. 5B). These data suggested that miR-30a-5p was important in RCC cell apoptosis.

GALNT7 is a direct target of miR-30a-5p. miRNAs exert their functions by regulating the expression of their downstream target gene/s. Multiple computational algorithms using miRanda, TargetScan, microRNA and miRWalk predicted that the 3'-UTR of the human GALNT7 gene is a target for miR-30a-5p. The 3'-UTR of GALNT7 mRNA contained a complementary nucleotide sequence for the seed region of miR-30a-5p (Fig. 6A). This seed region of miR-30a-5p was highly conserved among species, indicating its regulatory importance (Fig. 6B). As shown in Fig. 6C, the luciferase reporter assay confirmed that the activity of the reporter containing the 3'-UTR of GALNT7 was decreased significantly when the cells were transfected with the miR-30a-5p mimic in the 293T cells $(\mathrm{P}<0.01)$ and Hela cells $(\mathrm{P}<0.01)$, compared with those transfected with the NC. However, no significant changes were observed in the activity of the reporter
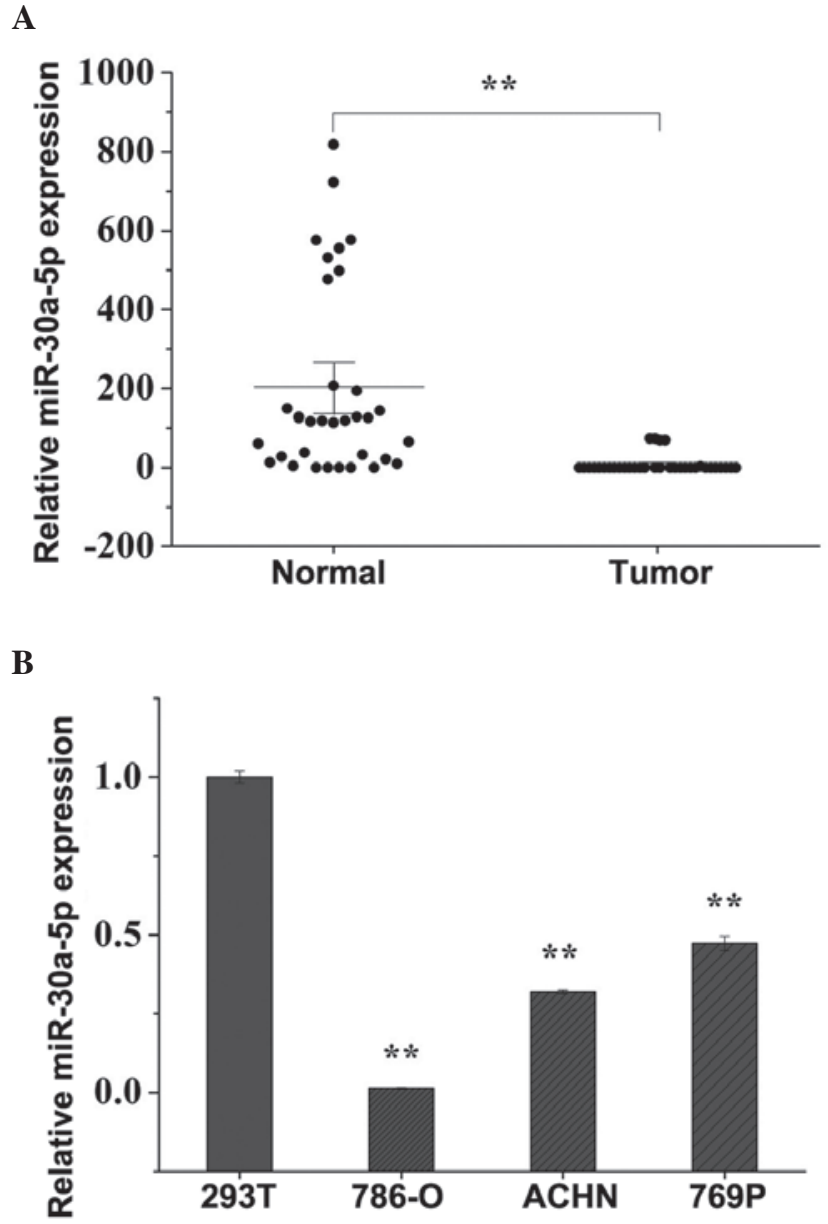

Figure 1. Expression of miR-30a-5p in RCC tissues and cell lines (A) Expression levels of miR-30a-5p relative to the expression levels of U6 were significantly lower in the $32 \mathrm{RCC}$ tumor samples, compared with the 32 paired adjacent nontumor tissues. (B) Reverse trancsription-quantitative polymerase chain reaction analysis showed that miR-30a-5p was downregulated significantly in the 786-O, ACHN and 769P cells, compared with the $293 \mathrm{~T}$ cells. The $2^{-\Delta \Delta \mathrm{Cq}}$ method was used to analyze data. Data are presented as the mean \pm standard deviation $\left({ }^{* *} \mathrm{P}<0.01\right)$. miR, microRNA; RCC, renal cell carcinoma.

containing the mutated seed sequence following transfection of the cells with either the miR-494 mimic or the NC.

miR-30a-5p downregulates the mRNA and protein levels of GALNT7 in RCC cell lines. To validate the results of the luciferase reporter assay and evaluate the association between miR-30a-5p and GALNT7, the present study performed RT-qPCR and Western blot analyses to quantify the mRNA and protein level of GALNT7 in the 786-O and ACHN cells $48 \mathrm{~h}$ following transfection with the miR-30a-5p mimic or NC. The mRNA levels of GALNT7 in the 786-O and ACHN cells were significantly decreased following transfection with the miR-30a-5p mimic, compared with the NC group $(\mathrm{P}<0.01$; Fig. 7A). In accordance with the downregulation in the mRNA levels of GALNT7, the protein levels of GALNT7 were significantly reduced when the 786-O and ACHN cells were transfected with the miR-30a-5p mimic ( $<<0.01$; Fig. 7B). These results suggested that miR-30a-5p regulated the mRNA and protein expression levels of GALNT7, and exerted regulation at transcriptional and post-transcriptional levels. 
A

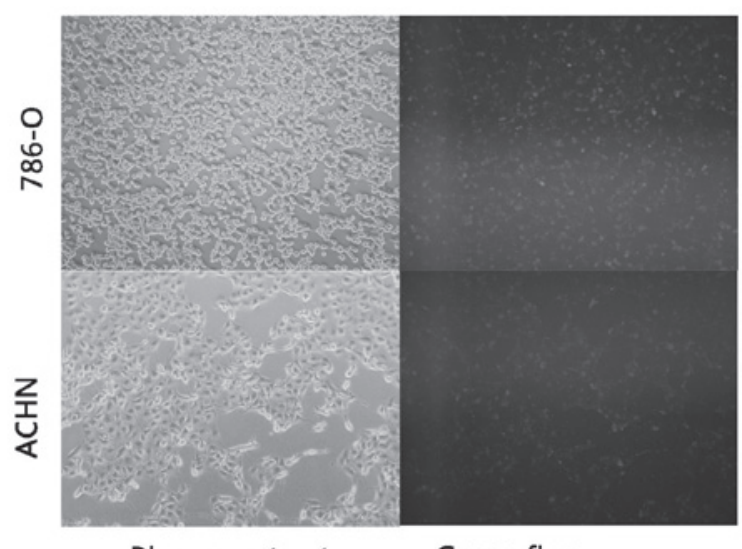

B

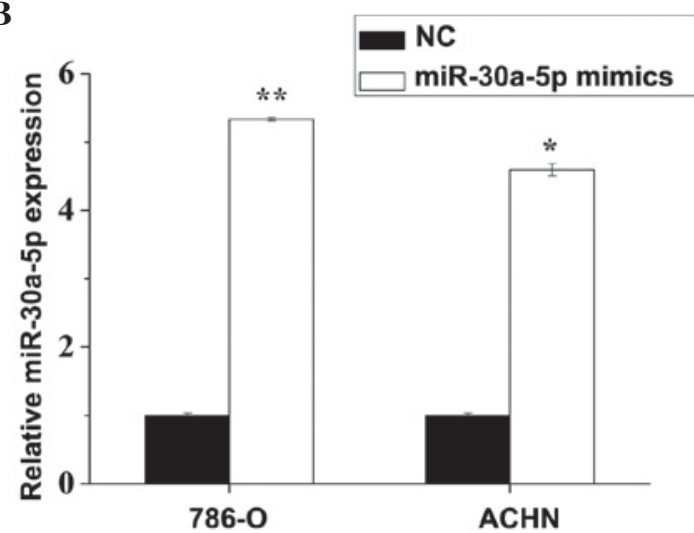

Figure 2. Confirmation of transient transfection efficiency. (A) Phase-contrast and green fluorescence images were captured from the same field (magnification, $\mathrm{x} 400$ ). (B) Relative expression levels of miR-30a-5p in the 786-O and ACHN cells transfected with the miR-30a-5p mimic or NC. Data are presented as the mean \pm standard deviation $\left({ }^{*} \mathrm{P}<0.05\right.$ and $\left.{ }^{* *} \mathrm{P}<0.01\right)$. miR, microRNA; NC, negative control.

A

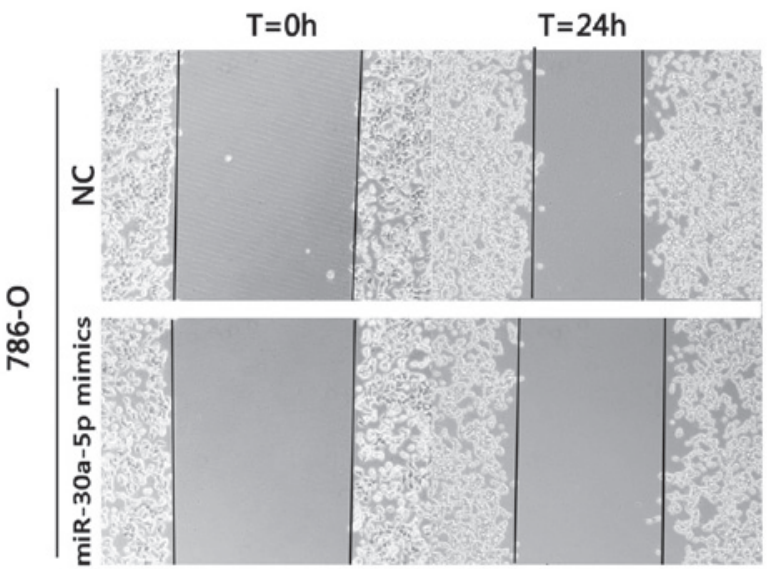

B

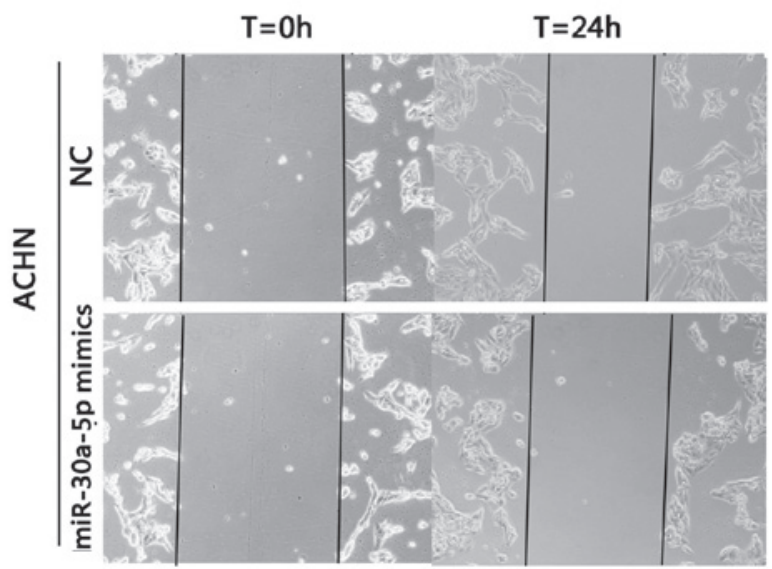

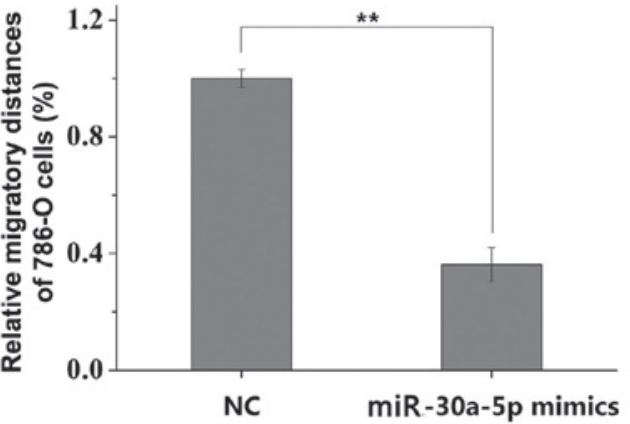

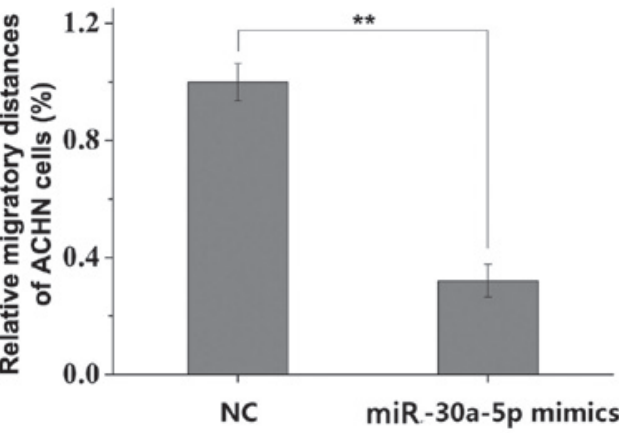

Figure 3. miR-30a-5p inhibits renal cell carcinoma cell migration. A wound scratch assay was performed to examine the migration of the (A) 786-O and (B) ACHN cells' following transfection with the miR-30a-5p mimic or NC. Data are presented as the mean \pm standard deviation $\left({ }^{* *} \mathrm{P}<0.01\right)$. miR, microRNA; $\mathrm{NC}$, negative control; T, time.

\section{Discussion}

miRNAs have multiple roles in various types of cancer, and the functions of miRNAs depend predominantly on their target genes, which include $\sim 50 \%$ of human protein-coding genes (9). Generally, the expression of functional miRNAs is dysregulated in various types of cancer, acting either as oncogenes when overexpressed, or tumor suppressors when downregulated $(31,32)$. The present study screened for differentially expressed miRNAs in RCC and confirmed that miR-30a-5p was markedly downregulated in RCC tissues and cell lines. The reduced expression levels of miR-30a-5p in 
A

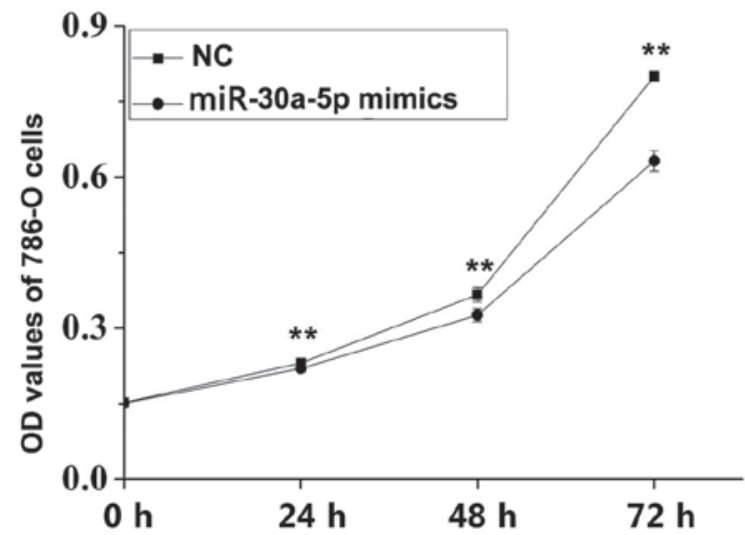

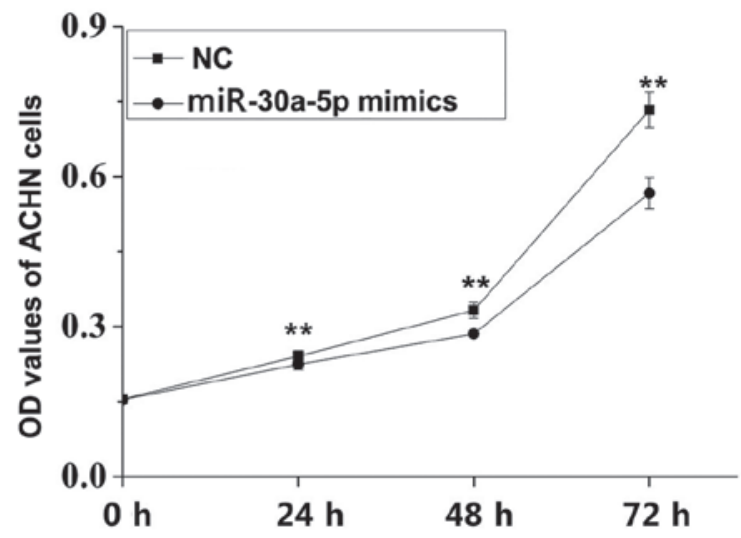

Figure 4. miR-30a-5p restrains renal cell carcinoma cell proliferation. Cell proliferation abilities of the (A) 786-O and (B) ACHN cells were measured using a 3-(4,5-dimethylthiazol-2-yl)-2,5-diphenyltetrazoliumbromide assay at 0,24, 48 and 72 h following transfection with miR-30a-5p mimic or NC. Data are presented as mean \pm standard deviation $\left({ }^{* *} \mathrm{P}<0.01\right)$. miR, microRNA; OD, optical density.

A

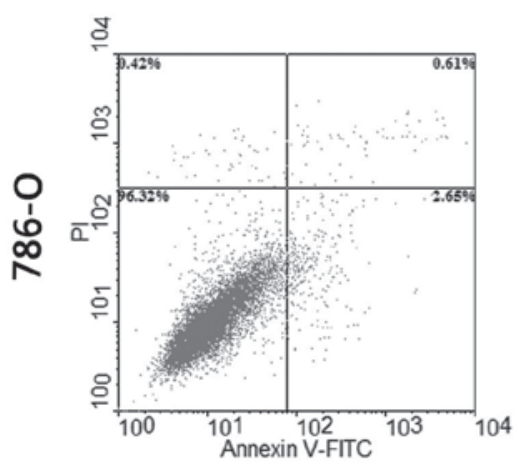

B I

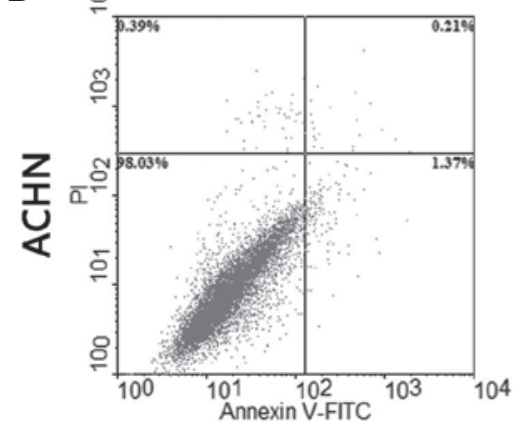

miR-30a-5p mimics
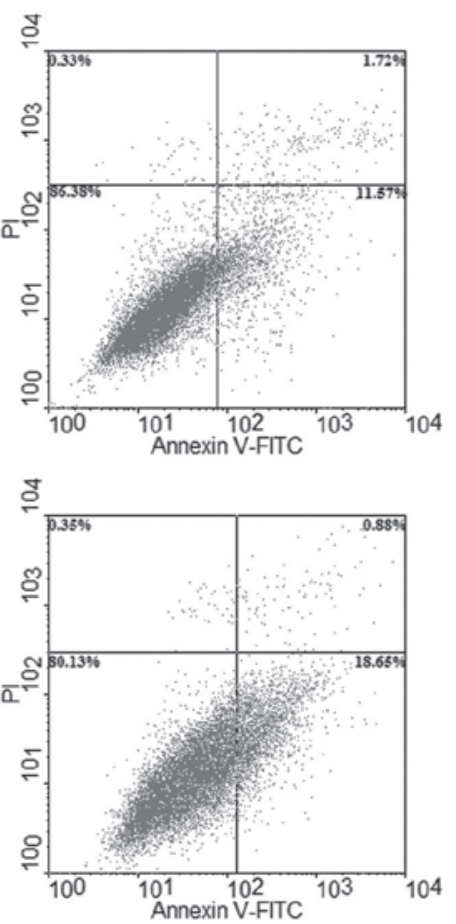
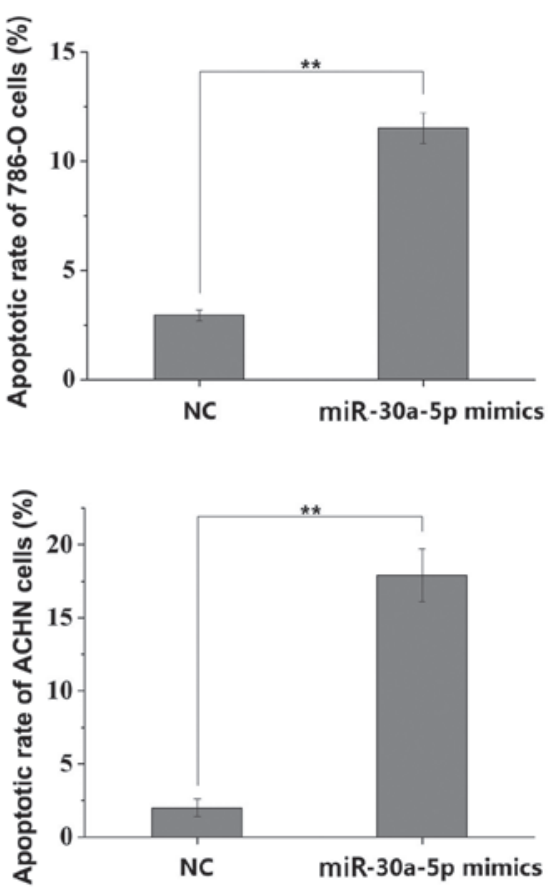

Figure 5. miR-30a-5p induces renal cell carcinoma cell apoptosis. Flow cytometry was used to measure the effect of miR-30a-5p on (A) 786-O and (B) ACHN cells, compared with the NC group. Data are presented as the mean \pm standard deviation $\left({ }^{* *} \mathrm{P}<0.01\right)$. miR, microRNA; NC, negative control; FITC, fluorescein isothiocyanate; PI, propidium iodide.

different types of cancer suggests that it may have anticancer effects. In the present study, the role of miR-30a-5p was investigated extensively, and its target gene in RCC cells was identified.

It has been reported that $\mathrm{miR}-30 \mathrm{a}-5 \mathrm{p}$ is important in the initiation and development of cancer. For example, miR-30a has tumor suppressive effects towards T-ALL and DLBCL cell lines by cell growth suppression, cell cycle arrest and the induction of apoptosis, whereas depletion of miR-30a restores tumorigenesis (33). In chronic myeloid leukemia, miR-30a decreases proliferation and arrests cell cycle progression between the G1 and S phases, acting as a tumor suppressor by downregulating the expression levels of abelson murine leukemia viral oncogene homolog 1 (ABL1) and breakpoint cluster protein-ABL1 (34). The dowregulation of miR-30a promotes cell survival and proliferation in T-cell lymphoblastic lymphomas via activation of GLI/Hedgehog signaling (35). Low expression of miR-30a in hepatocellular carcinoma facilitate migration, invasion and epithelial-mesenchymal transition by targeting SNAI1 (17). In addition, miR-30a promotes invasiveness and metastasis in vitro and in vivo through epithelial-mesenchymal transition, and results in poor survival 
$\mathbf{A}$
GALNT7 MUT 3' UTR
GALNT7 WT 3' UTR
hsa-mir-30a-5p
5' ... AAAAUUUUCAAAUAAACAAAUGC. .
$5{ }^{\prime}$... AUCAUCUGCAGUUACUGUUUACA. .
3' GAAGGUCAGCUCCUACAAAUGU

B

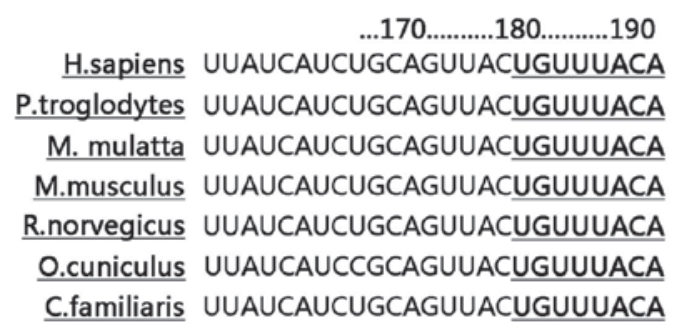

C

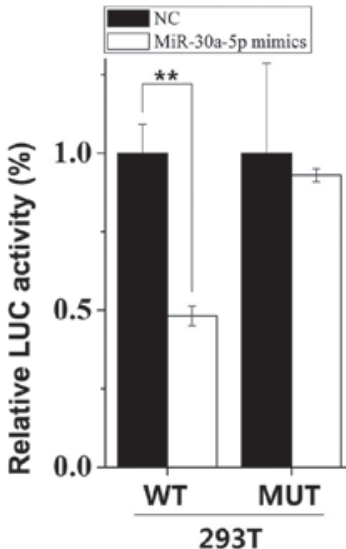

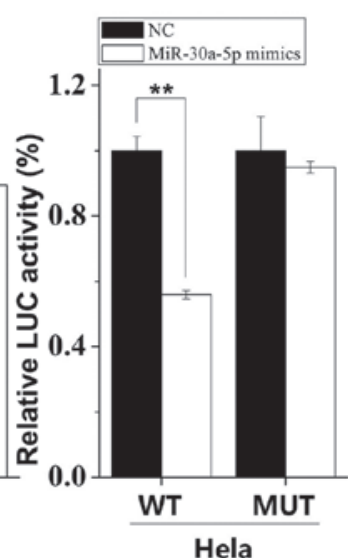

Figure 6. GALNT7 is targeted by miR-30a-5p. (A) A 519 bp fragment of GALNT7 mRNA was constructed, which contained the WT potential binding sites of miR-30a-5p and the mutated sequence (MUT). (B) Comparison of the miR-30a-5p seed sequence in seven species. (C) Results of a luciferase reporter assay for $293 \mathrm{~T}$ and Hela cells following co-transfection with the constructed plasmids and the miR-30a-5p mimic or NC. All luciferase assays were performed in triplicate and measurements were made $24 \mathrm{~h}$ following transfection. Data are presented as the mean \pm standard deviation $\left({ }^{* *} \mathrm{P}<0.01\right)$. miR, microRNA; WT, wild-type; MUT, mutant; LUC, luciferase.

A

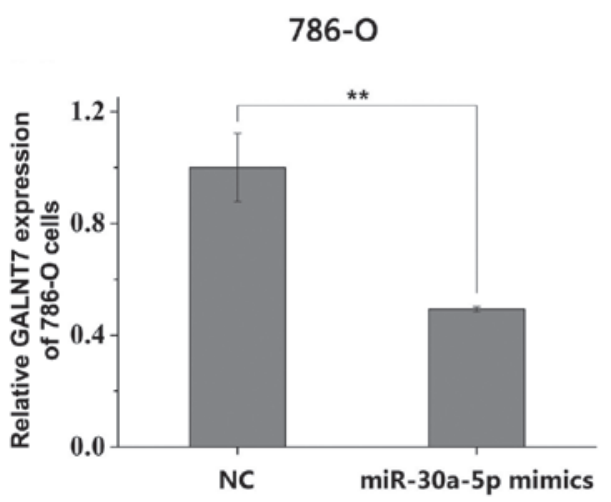

B

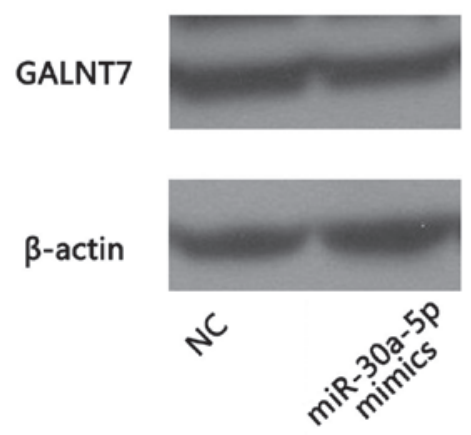

$\mathrm{ACHN}$

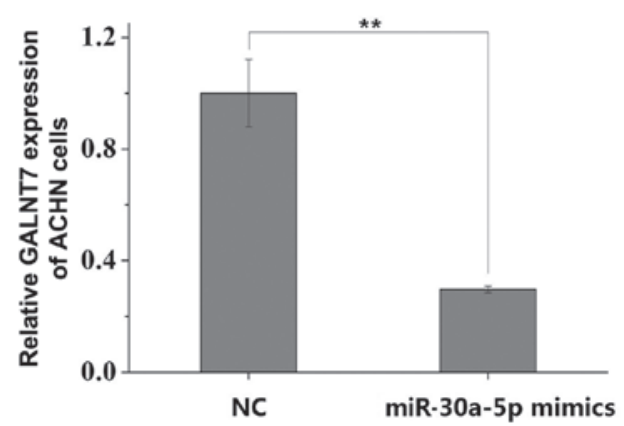

GALNT7

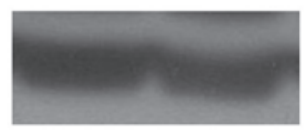

$\beta$-actin
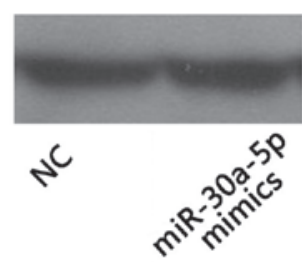

Figure 7. Regulation of GALNT7 by miR-30a-5p. The (A) mRNA and (B) protein levels of 786-O and ACHN cells were downregulated significantly following transfection with the miR-30a-5p mimic, compared with those transfected with $\mathrm{NC}\left({ }^{* *} \mathrm{P}<0.01\right)$. GALNT7, Polypeptide $\mathrm{N}$-acetylgalactosaminyltransferase 7 ; miR, microRNA; NC, negative control.

rates in patients with nasopharyngeal carcinoma (18). All the above studies indicate that downregulation of miR-30a occurs frequently in human cancer, and has functions in tumor onset and progression. However, its relevance in the development of
RCC remains to be fully elucidated. Accordingly, the present study revealed that the upregulation of miR-30a-5p inhibited the migration and proliferation abilities, and promoted the apoptosis of RCC cells. 
GALNT7 is a member of the GalNAc-transferase family, which is important in initiating mucin-type O-glycosylation in the Golgi apparatus by transferring GalNAc from UDP-GalNAc to the Ser and Thr residues of polypeptide acceptors (36). Mucin is a cell-surface glycoprotein, which establishes a molecular barrier at the epithelial surface and engages in morphogenetic signal transduction. Alterations in mucin glycosylation accompany the development of cancer and affect cellular growth, differentiation, transformation, adhesion, invasion and immune surveillance $(37,38)$. According to previous studies, GALNT7 is upregulated in several types of cancer, including laryngeal carcinoma, cervical cancer and pancreatic cancer (39-41), and is targeted by tumor suppressive miRNAs. Additionally, GALNT7, as a target of upregulated miR-30b/30d is important in the metastatic behavior of melanoma cells (42). Previously, the expression of GALNT7 in thyroid tissues, in relation to iodine-131 doses received from the Chernobyl accident, were investigated, and a statistically significant dose-expression association was confirmed, suggesting that GALNT7 is important in radiation carcinogenesis (43). In the present study, GALNT7 was identified as a downstream gene of miR-30a-5p following bioinformatics and experimental verification. However, the expression and function of GALNT7 in RCC remains to be fully elucidated, and its role warrants confirmation in future investigations.

It appears controversial that miR-30a-5p functions as a tumor suppressor in certain types of cancer, and an oncogene in others. Unlike small interfering RNA, the interactions between miRNAs and the 3'-UTRs of target genes are not entirely complementary (44). Due to the fact that the seed region between miRNAs and genes requires only $\geq 7$ base pairs, the network between miRNAs and target genes is not one-to-one, but one-to-multiple and multiple-to-one. Additionally, the expression of miRNAs was various in different tissues and stages of development, thus the functions of miRNAs are tissue-and temporally-specific.

In conclusion, the present study provided evidence indicating that miR-30a-5p may be an important tumor suppressor in RCC tumorigenesis by targeting GALNT7.

\section{Acknowledgements}

This work was supported by the National Natural Science Foundation of China (no. 81101922), the Guangdong Natural Science Foundation (no. 2015A030313889), Science and Technology Development Fund Project of Shenzhen (nos. JCYJ20120616144352139, JCYJ20130402114702124, JCYJ20140415162542975 and JCYJ20150403091443329) and the Fund of Guangdong Key Medical Subject.

\section{References}

1. Siegel R, Ma J, Zou Z and Jemal A: Cancer statistics, 2014. CA Cancer J Clin 64: 9-29, 2014.

2. Rini BI, Campbell SC and Escudier B: Renal cell carcinoma. Lancet 373: 1119-1132, 2009.

3. Motzer RJ, Bander NH and Nanus DM: Renal-cell carcinoma. N Engl J Med 335: 865-875, 1996.

4. Janzen NK, Kim HL, Figlin RA and Belldegrun AS: Surveillance after radical or partial nephrectomy for localized renal cell carcinoma and management of recurrent disease. Urol Clin North Am 30: 843-852, 2003.
5. Rouvière $\mathrm{O}$, Bouvier R, Négrier S, Badet L and Lyonnet D: Nonmetastatic renal-cell carcinoma: Is it really possible to define rational guidelines for post-treatment follow-up? Nat Clin Pract Oncol 3: 200-213, 2006.

6. Carthew RW and Sontheimer EJ: Origins and mechanisms of miRNAs and siRNAs. Cell 136: 642-655, 2009.

7. Huntzinger $\mathrm{E}$ and Izaurralde E: Gene silencing by microRNAs: Contributions of translational repression and mRNA decay. Nat Rev Genet 12: 99-110, 2011.

8. Bartel DP: MicroRNAs: Target recognition and regulatory functions. Cell 136: 215-233, 2009.

9. Krol J, Loedige I and Filipowicz W: The widespread regulation of microRNA biogenesis, function and decay. Nat Rev Genet 11: 597-610, 2010.

10. Ichimi T, Enokida H, Okuno Y, Kunimoto R, Chiyomaru T, Kawamoto K, Kawahara K, Toki K, Kawakami K, Nishiyama K, et al: Identification of novel microRNA targets based on microRNA signatures in bladder cancer. Int J Cancer 125: 345-352, 2009.

11. Rydzanicz M, Wrzesiński T, Bluyssen HA and Wesoly J: Genomics and epigenomics of clear cell renal cell carcinoma: Recent developments and potential applications. Cancer Lett 341: 111-126, 2013.

12. Fendler A, Stephan C, Yousef GM and Jung K: MicroRNAs as regulators of signal transduction in urological tumors. Clin Chem 57: 954-968, 2011.

13. Huang QB, Ma X, Zhang X, Liu SW, Ai Q, Shi TP, Zhang Y, Gao Y,Fan Y, Ni D, et al: Down-Regulated miR-30a in Clear Cell Renal Cell Carcinoma Correlated with Tumor Hematogenous Metastasis by Targeting Angiogenesis-Specific DLL4. PLoS One 8, 2013

14. Mathew LK, Lee SS, Skuli N, Rao S, Keith B, Nathanson KL, Lal P and Simon MC: Restricted expression of miR-30c-2-3p and miR-30a-3p in clear cell renal cell carcinomas enhances HIF2alpha activity. Cancer Discov 4: 53-60, 2014.

15. Tan X, Qin W, Zhang L, Hang J, Li B, Zhang C, Wan J, Zhou F, Shao K, Sun Y, et al: A 5-microRNA signature for lung squamous cell carcinoma diagnosis and hsa-miR-31 for prognosis. Clin Cancer Res 17: 6802-6811, 2011.

16. Fuster O, Llop M, Dolz S, García P, Such E, Ibáñez M, Luna I, Gómez I, López M, Cervera J, et al: Adverse prognostic value of MYBL2 overexpression and association with microRNA-30 family in acute myeloid leukemia patients. Leuk Res 37: 1690-1696, 2013.

17. Liu Z, Tu K and Liu Q: Effects of microRNA-30a on migration, invasion and prognosis of hepatocellular carcinoma. FEBS Lett 588: 3089-3097, 2014.

18. Wang HY, Li YY, Fu S, Wang XP, Huang MY, Zhang X, Shao Q, Deng L, Zeng MS, Zeng YX and Shao JY: MicroRNA-30a promotes invasiveness and metastasis in vitro and in vivo through epithelial-mesenchymal transition and results in poor survival of nasopharyngeal carcinoma patients. Exp Biol Med 239: 891-898, 2014.

19. Huang Q, Jiang Z, Meng T, Yin H, Wang J, Wan W, Cheng M, Yan W, Liu T, Song D, et al: MiR-30a inhibits osteolysis by targeting RunX2 in giant cell tumor of bone. Biochem Biophys Res Commun 453: 160-165, 2014.

20. Fu J, Xu X, Kang L, Zhou L, Wang S, Lu J, Cheng L, Fan Z, Yuan B, Tian P, et al: miR-30a suppresses breast cancer cell proliferation and migration by targeting Eya2. Biochem Biophys Res Commun 445: 314-319, 2014.

21. Jiang X, Du L, Wang L, Li J, Liu Y, Zheng G, Qu A, Zhang X, Pan H, Yang Y and Wang C: Serum microRNA expression signatures identified from genome-wide microRNA profiling serve as novel noninvasive biomarkers for diagnosis and recurrence of bladder cancer. Int J Cancer 136: 854-862, 2015.

22. Jia Z, Wang K, Wang G, Zhang A and Pu P: MiR-30a-5p antisense oligonucleotide suppresses glioma cell growth by targeting SEPT7. PLoS One 8: e55008, 2013.

23. Wang K, Jia Z, Zou J, Zhang A, Wang G, Hao J, Wang Y, Yang S and $\mathrm{Pu}$ P: Analysis of hsa-miR-30a-5p expression in human gliomas. Pathol Oncol Res 19: 405-411, 2013.

24. Liu H, Brannon AR, Reddy AR, Alexe G, Seiler MW, Arreola A, Oza JH, Yao M, Juan D, Liou LS, et al: Identifying mRNA targets of microRNA dysregulated in cancer: With application to clear cell renal cell carcinoma. BMC Syst Biol 4: 51, 2010.

25. Juan D, Alexe G, Antes T, Liu H, Madabhushi A, Delisi C, Ganesan S, Bhanot G and Liou LS: Identification of a microRNA panel for clear-cell kidney cancer. Urology 75 : $835-841,2010$. 
26. Yi Z, Fu Y, Zhao S, Zhang X and Ma C: Differential expression of miRNA patterns in renal cell carcinoma and nontumorous tissues. J Cancer Res Clin Oncol 136: 855-862, 2010.

27. White NM, Khella HW, Grigull J, Adzovic S, Youssef YM, Honey RJ, Stewart R, Pace KT, Bjarnason GA, Jewett MA, et al: miRNA profiling in metastatic renal cell carcinoma reveals a tumour-suppressor effect for miR-215. Br J Cancer 105: $1741-1749,2011$

28. Müller S and Nowak K: Exploring the miRNA-mRNA regulatory network in clear cell renal cell carcinomas by next-generation sequencing expression profiles. Biomed Res Int 2014: 948408, 2014.

29. Edge SB, Byrd DR, Compton CC, Fritz AG, Greene FL, Trotti A eds. AJCC Cancer Staging Manual. 7th ed., New York, NY: Springer, 479-489, 2010

30. Duan HF, Li XQ, Hu HY, Li YC, Cai Z, Mei XS, Yu P, Nie LP Zhang W, Yu ZD and Nie GH: Functional elucidation of miR-494 in the tumorigenesis of nasopharyngeal carcinoma. Tumour Biol 36: 6679-6689, 2015.

31. Shenouda SK and Alahari SK: MicroRNA function in cancer: Oncogene or a tumor suppressor? Cancer Metastasis Rev 28 : 369-378, 2009.

32. Garzon R, Calin GA and Croce CM: MicroRNAs in Cancer. Annu Rev Med 60: 167-179, 2009.

33. Ortega M, Bhatnagar H, Lin AP, Wang L, Aster JC, Sill H and Aguiar RC: A microRNA-mediated regulatory loop modulates NOTCH and MYC oncogenic signals in B-and T-cell malignancies. Leukemia 29: 968-976, 2015.

34. Liu Y, Song Y, Ma W, Zheng W and Yin H: Decreased microRNA-30a levels are associated with enhanced ABL1 and BCR-ABL1 expression in chronic myeloid leukemia. Leuk Res 37: 349-356, 2013.

35. González-Gugel E, Villa-Morales M, Santos J, Bueno MJ, Malumbres M, Rodríguez-Pinilla SM, Piris MÁ and Fernández-Piqueras J: Down-regulation of specific miRNAs enhances the expression of the gene Smoothened and contributes to T-cell lymphoblastic lymphoma development. Carcinogenesis 34: 902-908, 2013.
36. Perrine CL, Ganguli A, Wu P, Bertozzi CR, Fritz TA, Raman J, Tabak LA and Gerken TA: Glycopeptide-preferring polypeptide GalNAc transferase 10 (ppGalNAc T10), involved in mucin-type $\mathrm{O}$-glycosylation, has a unique GalNAc-O-Ser/Thr-binding site in its catalytic domain not found in ppGalNAc T1 or T2. J Biol Chem 284: 20387-20397, 2009.

37. Pegram MD, Borges VF, Ibrahim N, Fuloria J, Shapiro C, Perez S, Wang K, Schaedli Stark F and Courtenay Luck N: Phase I dose escalation pharmacokinetic assessment of intravenous humanized anti-MUC1 antibody AS1402 in patients with advanced breast cancer. Breast Cancer Res 11: R73, 2009.

38. Hollingsworth MA and Swanson BJ: Mucins in cancer: Protection and control of the cell surface. Nat Rev Cancer 4: 45-60, 2004

39. Peng RQ, Wan HY, Li HF, Liu M, Li $X$ and Tang H: MicroRNA-214 suppresses growth and invasiveness of cervical cancer cells by targeting UDP-N-acetyl- $\alpha$-D-galactosamine: Polypeptide N-acetylgalactosaminyltransferase 7. J Biol Chem 287: 14301-14309, 2012.

40. Taniuchi K, Cerny RL, Tanouchi A, Kohno K, Kotani N, Honke K, Saibara T and Hollingsworth MA: Overexpression of GalNAc-transferase GalNAc-T3 promotes pancreatic cancer cell growth. Oncogene 30: 4843-4854, 2011.

41. Li W, Ma H and Sun J: MicroRNA34a/c function as tumor suppressors in Hep2 laryngeal carcinoma cells and may reduce GALNT7 expression. Mol Med Rep 9: 1293-1298, 2014.

42. Gaziel-Sovran A, Segura MF, Di Micco R, Collins MK, Hanniford D, Vega-Saenz de Miera E, Rakus JF, Dankert JF, Shang S, Kerbel RS, et al: miR-30b/30d regulation of GalNAc transferases enhances invasion and immunosuppression during metastasis. Cancer Cell 20: 104-118, 2011.

43. Abend M, Pfeiffer RM, Ruf C, Hatch M, Bogdanova TI, Tronko MD, Riecke A, Hartmann J, Meineke V, Boukheris $\mathrm{H}$, et al: Iodine-131 dose dependent gene expression in thyroid cancers and corresponding normal tissues following the Chernobyl accident. PLoS One 7: e39103, 2012.

44. Kim VN: MicroRNA biogenesis: Coordinated cropping and dicing. Nat Rev Mol Cell Biol 6: 376-385, 2005. 